\title{
ON WEAK SOLUTIONS TO THE EQUATIONS OF NON-STATIONARY MOTION OF HEAT-CONDUCTING INCOMPRESSIBLE VISCOUS FLUIDS: DEFECT MEASURE AND ENERGY EQUALITY
}

\author{
JOACHIM NAUMANN \\ Institut für Mathematik, Humboldt-Universität zu Berlin \\ Unter den Linden 6, 10099 Berlin, Germany \\ E-mail: jnaumann@mathematik.hu-berlin.de
}

\begin{abstract}
We consider the non-stationary Navier-Stokes equations completed by the equation of conservation of internal energy. The viscosity of the fluid is assumed to depend on the temperature, and the dissipation term is the only heat source in the conservation of internal energy. For the system of PDE's under consideration, we prove the existence of a weak solution such that: 1) the weak form of the conservation of internal energy involves a defect measure, and 2) the equality for the total energy is satisfied.
\end{abstract}

1. Introduction. Let $\Omega \subset \mathbf{R}^{3}$ be a bounded domain, and let $0<T<\infty$. In $Q=$ $\Omega \times] 0, T[$ we consider the following system of PDE's:

$$
\begin{aligned}
\nabla \cdot \mathbf{u} & =0 \\
\frac{\partial \mathbf{u}}{\partial t}+(\mathbf{u} \cdot \nabla) \mathbf{u} & =\nabla \cdot(\nu(\theta) D(\mathbf{u}))-\nabla p+\mathbf{f}, \\
\frac{\partial \theta}{\partial t}+\mathbf{u} \cdot \nabla \theta & =\nu(\theta) D(\mathbf{u}): D(\mathbf{u})-\nabla \cdot(\kappa(\theta) \nabla \theta),
\end{aligned}
$$

where $\mathbf{u}=\left(u_{1}, u_{2}, u_{3}\right)=$ velocity, $p=$ pressure, $\mathbf{f}=$ external force $\theta=$ temperature, $D(\mathbf{u})=\frac{1}{2}\left(\nabla \mathbf{u}+(\nabla \mathbf{u})^{\top}\right)=$ rate of strain tensor, $\nu(\theta)=$ viscosity, $\kappa(\theta)=$ heat conductivity.

Equations (1.1)-(1.3) represent the laws of conservation of mass, momentum and internal enery, respectively, of an incompresible fluid in non-stationary motion, where

2000 Mathematics Subject Classification: Primary 35Q30; Secondary 76D05.

Key words and phrases: Navier-Stokes equations, existence of weak solutions.

The paper is in final form and no version of it will be published elsewhere. 
the temperature $\theta$ stands for the internal energy. The term $\nu(\theta) D(\mathbf{u}): D(\mathbf{u})^{1}$ on the right hand side of (1.3) models the dissipation of internal energy due to viscosity (cf. [3], [4], [5], [7], [14] for more details).

We complete (1.1)-(1.3) by the following boundary and initial conditions on $\mathbf{u}$ and $\theta$ :

$$
\begin{aligned}
& \left.\mathbf{u}=0, \quad \frac{\partial \theta}{\partial n}=0 \quad \text { on } \partial \Omega \times\right] 0, T[, \\
& \mathbf{u}=\mathbf{u}_{0}, \quad \theta=\theta_{0} \quad \text { on } \Omega \times\{0\},
\end{aligned}
$$

where $n$ denotes the unit outward normal along $\partial \Omega$.

The aim of the present paper is to continue the discussions of (1.1)-(1.5) we began in [12], by a more detailed study of the weak solution and the associated defect measure obtained there.

2. Notations. Existence of an approximate solution. Without any further reference, throughout the paper the boundary $\partial \Omega$ is assumed to be Lipschitzian.

Let $W^{1, q}(\Omega)(1 \leq q<\infty)$ denote the usual Sobolev space. Define

$$
\begin{aligned}
& V_{q}:=\left\{\mathbf{v} \in W^{1, q}\left(\Omega ; \mathbf{R}^{3}\right) \mid \mathbf{v}=0 \text { a.e. on } \partial \Omega, \operatorname{div} \mathbf{v}=0 \text { a.e. in } \Omega\right\}, \\
& H:=\left\{\mathbf{h} \in L^{2}\left(\Omega ; \mathbf{R}^{3}\right) \mid \int_{\Omega} \mathbf{h} \cdot \nabla \varphi d x=0 \forall \varphi \in C_{c}^{\infty}(\Omega)\right\} .
\end{aligned}
$$

$V_{q}$ is a Banach space with respect to the norm

$$
\|\mathbf{v}\|_{V_{q}}:=\left(\sum_{i, j=1}^{3} \int_{\Omega}\left|\frac{\partial v_{i}}{\partial x_{j}}\right|^{q} d x\right)^{1 / q}
$$

$H$ is a Hilbert space with respect to the scalar product

$$
(\mathbf{g}, \mathbf{h})_{H}:=\int_{\Omega} \mathbf{g} \cdot \mathbf{h} d x .
$$

The following imbeddings are readily established:

$$
\begin{gathered}
V_{q} \subset H \quad \text { continuously, densely if } q \geq 6 / 5, \\
V_{q} \subset H \quad \text { compactly if } \quad q>6 / 5
\end{gathered}
$$

(cf. [12] for details).

Next, given a normed space $X$ with norm $\|\cdot\|$, we denote by $X^{*}$ its dual space, by $\|\cdot\|_{*}$ the dual norm on $X^{*}$ and by $\left\langle z^{*}, z\right\rangle_{X}$ the value of the continuous linear functional $z^{*} \in X^{*}$ at $z \in X$.

By $L^{q}(0, T ; X)(1 \leq q \leq \infty)$ we denote the vector space of all equivalence classes of Bochner measurable functions $z:[0, T] \rightarrow X$ such that

$$
\int_{0}^{T}\|z(t)\|^{q} d t<\infty \quad \text { if } 1 \leq q<\infty, \quad \underset{t \in[0, T]}{\operatorname{ess} \sup }\|z(t)\|<\infty \quad \text { if } q=\infty .
$$

Finally, let $\mathcal{M}(\bar{Q})$ denote the space of all Radon measures in $\bar{Q}$.

\footnotetext{
${ }^{1}$ Given two matrices $A=\left\{A_{i j}\right\}, B=\left\{B_{i j}\right\}$, the trace of their product is defined by $A: B=$ $A_{i j} B_{i j}$. Throughout the paper, repeated Latin subscripts imply summation on $1,2,3$.
} 
For notational simplicity, in what follows we write $L^{q}$ and $W^{1, q}$ in place of $L^{q}(\Omega)$ and $W^{1, q}(\Omega)$, respectively. Define

$$
D(\mathbf{u}, \mathbf{v}):=D_{i j}(\mathbf{u}) D_{i j}(\mathbf{v}) .
$$

The following result has been proved in [13].

Theorem 1. Assume $\mathbf{u}_{0} \in V_{6}, \theta_{0} \in W^{1,2}$. Fix $\left.r \in\right] 6 / 5,5 / 4[$, and define $\rho:=8 r /(5 r-6)$. Then for every $\varepsilon>0$ there exists $\left\{\mathbf{u}_{\varepsilon}, \theta_{\varepsilon}\right\}$ such that

$$
\left\{\begin{array}{l}
\mathbf{u}_{\varepsilon} \in L^{2}\left(0, T ; V_{2}\right) \cap C([0, T] ; H), \mathbf{u}_{\varepsilon}^{\prime} \in L^{2}\left(0, T ; V_{2}^{*}\right), \\
\theta_{\varepsilon} \in L^{r}\left(0, T ; W^{1, r}\right) \cap L^{\infty}\left(0, T ; L^{1}\right) \cap C\left([0, T] ;\left(W^{1, \rho}\right)^{*}\right), \\
\theta_{\varepsilon}^{\prime} \in L^{1}\left(0, T ;\left(W^{1, \rho}\right)^{*}\right),
\end{array}\right.
$$

and

$$
\begin{aligned}
\int_{0}^{T}\left\langle\mathbf{u}_{\varepsilon}^{\prime}, \mathbf{v}\right\rangle_{V_{2}} d t+\int_{Q} \nu\left(\theta_{\varepsilon}\right) D\left(\mathbf{u}_{\varepsilon}, \mathbf{v}\right) d x d t-\int_{Q} & u_{\varepsilon i} u_{\varepsilon j} \Phi_{\varepsilon}\left(\left|\mathbf{u}_{\varepsilon}\right|^{2}\right) \frac{\partial v_{i}}{\partial x_{j}} d x d t \\
& =\int_{0}^{T}\langle\mathbf{f}, \mathbf{v}\rangle_{V_{2}} d t \forall \mathbf{v} \in L^{2}\left(0, T ; V_{2}\right)
\end{aligned}
$$

$$
\begin{gathered}
\int_{0}^{T}\left\langle\theta_{\varepsilon}^{\prime}, \varphi\right\rangle_{W^{1, \rho}} d t-\int_{Q} u_{\varepsilon i} \theta_{\varepsilon} \frac{\partial \varphi}{\partial x_{i}} d x d t+\int_{Q} \kappa\left(\theta_{\varepsilon}\right) \nabla \theta_{\varepsilon} \cdot \nabla \varphi d x d t \\
=\int_{Q} \nu\left(\theta_{\varepsilon}\right) D\left(\mathbf{u}_{\varepsilon}, \mathbf{u}_{\varepsilon}\right) \varphi d x d t \forall \varphi \in L^{\infty}\left(0, T ; W^{1, \rho}\right), \\
\mathbf{u}_{\varepsilon}(0)=\mathbf{u}_{0}, \quad \theta_{\varepsilon}(0)=\theta_{0} .
\end{gathered}
$$

In addition,

$$
\begin{aligned}
& \frac{1}{2} \int_{\Omega}\left|\mathbf{u}_{\varepsilon}(t)\right|^{2} d x+\int_{0}^{t} \int_{\Omega} \nu\left(\theta_{\varepsilon}\right) D\left(\mathbf{u}_{\varepsilon}, \mathbf{u}_{\varepsilon}\right) d x d s \\
& \qquad=\frac{1}{2} \int_{\Omega}\left|\mathbf{u}_{0}\right|^{2} d x+\int_{0}^{t}\left\langle\mathbf{f}, \mathbf{u}_{\varepsilon}\right\rangle_{V_{2}} d s \forall t \in[0, T], \\
& \left\{\begin{array}{l}
\left\|\mathbf{u}_{\varepsilon}^{\prime}\right\|_{L^{4 / 3}\left(0, T ; V_{2}^{*}\right)} \leq c\left(1+\left\|\mathbf{u}_{0}\right\|_{H}+\|\mathbf{f}\|_{L^{2}\left(0, T ; V_{2}^{*}\right)}\right)\left(\left\|\mathbf{u}_{0}\right\|_{H}+\|\mathbf{f}\|_{L^{2}\left(0, T ; V_{2}^{*}\right)}\right), \\
c=\text { const independent of } \varepsilon,
\end{array}\right. \\
& \operatorname{esssup} \int_{\Omega}\left|\theta_{\varepsilon}(t)\right| d x+\int_{Q}\left|\nabla \theta_{\varepsilon}\right|^{r} d x d t+\int_{Q} \frac{\left|\nabla \theta_{\varepsilon}\right|^{2}}{\left(1+\left|\theta_{\varepsilon}\right|\right)^{1+\sigma}} d x d t \leq C_{1}
\end{aligned}
$$

for all $0<\sigma<(5-4 r) / 3$ and

$$
\left\|\theta_{\varepsilon}^{\prime}\right\|_{L^{1}\left(0, T ;\left(W^{1, \rho}\right)^{*}\right)} \leq C_{2},
$$

where $C_{1}$ and $C_{2}$ are constants which depend on $\sigma, \operatorname{mes}(\Omega),\|\mathbf{f}\|_{L^{2}\left(0, T ; V_{2}^{*}\right)},\left\|\mathbf{u}_{0}\right\|_{H}$ and $\left\|\theta_{0}\right\|_{L^{1}}$, but are independent of $\varepsilon$. Moreover,

$$
\begin{aligned}
\int_{\Omega}\left(\frac{1}{2}\left|\mathbf{u}_{\varepsilon}(t)\right|^{2}\right. & \left.+\theta_{\varepsilon}(t)\right) d x \\
& =\int_{\Omega}\left(\frac{1}{2}\left|\mathbf{u}_{0}\right|^{2}+\theta_{0}\right) d x+\int_{0}^{t}\left\langle\mathbf{f}, \mathbf{u}_{\varepsilon}\right\rangle_{V_{2}} \text { ds for a.e. } t \in[0, T] .
\end{aligned}
$$


Here, the function $\Phi_{\varepsilon}$ in (2.4) is defined as follows. Fix a non-increasing function $\Phi \in C([0,+\infty[)$ such that

- $0 \leq \Phi \leq 1$ in $[0,+\infty[$,

- $\Phi=1$ in $[0,1], \Phi=0$ in $[2,+\infty[$.

Then, for $\varepsilon>0$ define

$$
\Phi_{\varepsilon}(\xi)=\Phi(\varepsilon \xi), \quad \xi \in[0,+\infty[.
$$

Clearly,

$$
\xi \Phi_{\varepsilon}(\xi) \leq \frac{2}{\varepsilon} \forall \xi \in\left[0,+\infty\left[, \forall \varepsilon>0, \lim _{\varepsilon \rightarrow 0} \Phi_{\varepsilon}(\xi)=1 \forall \xi \in[0,+\infty[\right.\right.
$$

3. Existence of a weak solution. We have the following

Theorem 2. Let $\mathbf{u}_{0} \in H, \theta_{0} \in L^{1}$. Then there exists a triple $\{\mathbf{u}, \theta, \mu\}$ such that

$$
\left\{\begin{array}{l}
\mathbf{u} \in C_{w}([0, T] ; H) \cap L^{2}\left(0, T ; V_{2}\right), \quad \mathbf{u}^{\prime} \in L^{4 / 3}\left(0, T ; V_{2}^{*}\right), \\
\theta \in L^{\infty}\left(0, T ; L^{1}(\Omega)\right) \cap \bigcap_{1 \leq r<\frac{5}{4}} L^{r}\left(0, T ; W^{1, r}(\Omega)\right), \\
\mu \in \mathcal{M}(\bar{Q})
\end{array}\right.
$$

and

$$
\begin{gathered}
\int_{0}^{T}\left\langle\mathbf{u}^{\prime}(t), \mathbf{v}(t)\right\rangle_{V_{2}} d t+\int_{Q} \nu(\theta) D(\mathbf{u}, \mathbf{v}) d x d t-\int_{Q} u_{i} u_{j} \frac{\partial v_{i}}{\partial x_{j}} d x d t \\
=\int_{0}^{T}\langle\mathbf{f}(t), \mathbf{v}(t)\rangle_{V_{2}} d t \forall \mathbf{v} \in L^{4}\left(0, T ; V_{2}\right), \\
-\int_{Q} \theta \frac{\partial \varphi}{\partial t} d t-\int_{Q} u_{i} \theta \frac{\partial \varphi}{\partial x_{i}} d x d t+\int_{Q} \kappa(\theta) \nabla \theta \cdot \nabla \varphi d x d t \\
=\int_{Q} \theta_{0}(x) \varphi(x, 0) d x+\int_{Q} \nu(\theta) D(\mathbf{u}, \mathbf{u}) \varphi d x d t+\int_{\bar{Q}} \varphi d \mu \forall \varphi \in C^{1}(\bar{Q}), \varphi(\cdot, T)=0, \\
\mathbf{u}(0)=\mathbf{u}_{0}, \quad \lim _{t \rightarrow 0} \int_{\Omega} \theta(x, t) d x=\int_{\Omega} \theta_{0}(x) d x
\end{gathered}
$$

In addition,

$$
\begin{gathered}
\frac{1}{2} \int_{\Omega}|\mathbf{u}(t)|^{2} d x+\int_{0}^{t} \int_{\Omega} \nu(\theta) D(\mathbf{u}, \mathbf{u}) d x d s \leq \frac{1}{2} \int_{\Omega}\left|\mathbf{u}_{0}\right|^{2} d x+\int_{0}^{t}\langle\mathbf{f}, \mathbf{u}\rangle_{V_{2}} d s \forall t \in[0, T], \\
\quad \underset{t \in[0, T]}{\operatorname{ess} \sup _{\Omega}} \int_{\Omega}|\theta(x, t)| d x+\int_{Q}|\nabla \theta|^{r} d x d t+\int_{Q} \frac{|\nabla \theta|^{2}}{(1+|\theta|)^{1+\sigma}} d x d t \leq c,
\end{gathered}
$$

for all $1 \leq r<5 / 4$ and $0<\sigma<(5-4 r) / 3$, where $c=$ const depends on $\|\mathbf{f}\|_{L^{2}\left(0, T ; V_{2}^{*}\right)}$, $\left\|\mathbf{u}_{0}\right\|_{H},\left\|\theta_{0}\right\|_{L^{1}}$ and $\sigma$, and

$$
\begin{aligned}
\int_{\Omega}\left(\frac{1}{2}|\mathbf{u}(x, t)|^{2}+\theta(x, t)\right) & d x \\
& =\int_{\Omega}\left(\frac{1}{2}\left|\mathbf{u}_{0}(x)\right|^{2}+\theta_{0}(x)\right) d x+\int_{0}^{t}\langle\mathbf{f}, \mathbf{u}\rangle_{V_{2}} d s \quad \text { for a.e. } t \in[0, T] .
\end{aligned}
$$


Here, $\mathbf{u}^{\prime}$ denotes the derivative of $\mathbf{u}$ in the sense of distributions on $] 0, T[$ with values in $V_{2}^{*}$.

Remarks. 1 . By $C_{w}([0, T] ; H)$ we denote the vector space of all mappings $\mathbf{v}:[0, T] \rightarrow H$ such that, for every $\mathbf{h} \in H$, the function $t \mapsto(\mathbf{v}(t), \mathbf{h})_{H}$ is continuous on $[0, T]$. We note that $\mathbf{u} \in L^{\infty}(0, T ; H)$ (cf. (3.5)) and $\mathbf{u}^{\prime} \in L^{4 / 3}\left(0, T ; V_{2}^{*}\right)$ imply the existence of a $\tilde{\mathbf{u}} \in C_{w}([0, T] ; H)$ such that $\tilde{\mathbf{u}}(t)=\mathbf{u}(t)$ for a.e. $t \in[0, T]$. Thus (3.5) has to be understood with $\tilde{\mathbf{u}}$ in place of $\mathbf{u}$.

Clearly, for every $\mathbf{v} \in C_{w}([0, T] ; H)$,

$$
\|\mathbf{v}(t)\|_{H} \leq \liminf _{s \rightarrow t}\|\mathbf{v}(s)\|_{H} \forall t \in[0, T] .
$$

From (3.5) it follows that

$$
\limsup _{t \rightarrow 0}\|\tilde{\mathbf{u}}(t)\|_{H} \leq\left\|\mathbf{u}_{0}\right\|_{H}
$$

Therefore $\lim _{t \rightarrow 0}\left\|\tilde{\mathbf{u}}(t)-\mathbf{u}_{0}\right\|_{H}=0$, i. e. $\tilde{\mathbf{u}}(0)=\mathbf{u}_{0}$ (cf. (3.4)).

2. Equation (3.7) implies that

$$
t \mapsto \int_{\Omega}\left(\frac{1}{2}|\mathbf{u}(x, t)|^{2}+\theta(x, t)\right) d x
$$

can be identified with an absolutely continuous function on $[0, T]$. In this sense, possible jump discontinuities of $\|\tilde{\mathbf{u}}(\cdot)\|_{H}^{2}=\int_{\Omega}|\mathbf{u}(x, \cdot)|^{2} d x$ are compensated by $2 \int_{\Omega} \theta(x, \cdot) d x$.

3. In addition to $\theta_{0} \in L^{1}(\Omega)$, assume $\operatorname{essinf}_{\Omega} \theta_{0} \geq 0$. Then

$$
\theta(x, t) \geq \underset{\Omega}{\operatorname{essinf}} \theta_{0} \quad \text { for a.e. }(x, t) \in Q .
$$

Proof of Theorem 2. Let $\left(\mathbf{u}_{0 \varepsilon}\right) \subset V_{6}$ and $\left(\theta_{0 \varepsilon}\right) \subset W^{1,2}(\varepsilon>0)$ be sequences such that $\mathbf{u}_{0 \varepsilon} \rightarrow \mathbf{u}_{0}$ strongly in $H$ and $\theta_{0 \varepsilon} \rightarrow \theta_{0}$ strongly in $L^{1}$ as $\varepsilon \rightarrow 0$.

Let $\left\{\mathbf{u}_{\varepsilon}, \theta_{\varepsilon}\right\}$ denote a pair obtained in Theorem 1 above. From (2.7), (2.8) and (2.9), (2.10) it follows that there exists a subsequence of $\left\{\mathbf{u}_{\varepsilon}, \theta_{\varepsilon}\right\}$ (not relabelled) such that

$$
\begin{array}{ll}
\mathbf{u}_{\varepsilon} \rightarrow \mathbf{u} & \text { weakly in } L^{2}\left(0, T ; V_{2}\right), \text { strongly in }\left[L^{2}(Q)\right]^{3}, \\
\mathbf{u}_{\varepsilon} \rightarrow \mathbf{u} & \text { a.e. in } Q, \\
\mathbf{u}_{\varepsilon} \rightarrow \mathbf{u} & \text { weakly in } L^{4 / 3}\left(0, T ; V_{2}^{*}\right)
\end{array}
$$

and

$$
\begin{aligned}
& \theta_{\varepsilon} \rightarrow \theta \text { weakly in } L^{r}\left(0, T ; W^{1, r}\right) \text {, weakly in } L^{4 r / 3}(Q) \text {, strongly in } L^{r}(Q), \\
& \theta_{\varepsilon} \rightarrow \theta \text { a.e. in } Q
\end{aligned}
$$

as $\varepsilon \rightarrow 0\left(1 \leq r<5 / 4\right.$; cf. [11], [12]). These convergence properties of $\left\{\mathbf{u}_{\varepsilon}, \theta_{\varepsilon}\right\}$ make it easy to pass to the limit $\varepsilon \rightarrow 0$ in (2.4) and (2.7). This gives (3.2) and (3.5) for a.e. $t \in[0, T]$, respectively. To see that (3.5) in fact holds for all $t \in[0, T]$, let $t \in[0, T]$. Then there exists a sequence $\left(t_{k}\right) \subset[0, T]$ such that $t_{k} \rightarrow t$ as $k \rightarrow \infty$, and (3.5) is true with $t_{k}$ in place of $t$. For the representative $\tilde{\mathbf{u}} \in C_{w}([0, T] ; H)$ of $\mathbf{u}$ we obtain

$$
\|\tilde{\mathbf{u}}(t)\|_{H} \leq \lim _{k \rightarrow \infty}\left\|\tilde{\mathbf{u}}\left(t_{k}\right)\right\|_{H}
$$


The first term on the left of (3.5) thus has to be understood with $\tilde{\mathbf{u}}$ in place of $\mathbf{u}$. In particular,

$$
\tilde{\mathbf{u}}(0)=\mathbf{u}_{0}(\operatorname{cf} .(3.4)), \quad \lim _{t \rightarrow 0}\|\tilde{\mathbf{u}}(t)\|_{H}=\left\|\mathbf{u}_{0}\right\|_{H} .
$$

To let $\varepsilon \rightarrow 0$ in (2.11) we multiply each term of (2.11) by $\zeta \in C_{c}^{\infty}(] 0, T[)$ and integrate over $[0, T]$. Observing that

$$
\begin{aligned}
\lim _{\varepsilon \rightarrow 0} \int_{Q}\left|\mathbf{u}_{\varepsilon}(x, t)\right|^{2} \zeta(t) d x d t & =\int_{Q}|\mathbf{u}(x, t)|^{2} \zeta(t) d x d t, \\
\lim _{\varepsilon \rightarrow 0} \int_{Q} \theta_{\varepsilon}(x, t) \zeta(t) d x d t & =\int_{Q} \theta(x, t) \zeta(t) d x d t,
\end{aligned}
$$

we find

$$
\int_{0}^{T} \zeta(t) \int_{\Omega}\left(\frac{1}{2}|\mathbf{u}(t)|^{2}+\theta(t)\right) d x d t=\int_{0}^{T} \zeta(t)\left\{\int_{\Omega}\left(\frac{1}{2}\left|\mathbf{u}_{0}\right|^{2}+\theta_{0}\right) d x+\int_{0}^{t}\langle\mathbf{f}, \mathbf{u}\rangle_{V_{2}} d s\right\} .
$$

This implies (3.7). Now, from (3.7) it follows that

$$
\lim _{t \rightarrow 0} \int_{\Omega} \theta(x, t) d x=\int_{\Omega} \theta_{0}(x) d x .
$$

Next, to let $\varepsilon \rightarrow 0$ in (2.5) we identify each function $\nu\left(\theta_{\varepsilon}\right) D\left(\mathbf{u}_{\varepsilon}, \mathbf{u}_{\varepsilon}\right)$ with a Radon measure in $\mathcal{M}(\bar{Q})$ to obtain a bounded sequence of measures. By weak* compactness of bounded sequences of measures, there exists a subsequence of $\left\{\mathbf{u}_{\varepsilon}, \theta_{\varepsilon}\right\}$ (not relabelled) and a Radon measure $\mu_{0} \in \mathcal{M}(\bar{Q})$ such that

$$
\lim _{\varepsilon \rightarrow 0} \int_{Q} \nu\left(\theta_{\varepsilon}\right) D\left(\mathbf{u}_{\varepsilon}, \mathbf{u}_{\varepsilon}\right) \varphi d x d t=\int_{\bar{Q}} \varphi d \mu_{0} \forall \varphi \in C(\bar{Q}) .
$$

Integrating by parts the first term on the left of (2.5) and letting $\varepsilon \rightarrow 0$ gives

$$
\begin{aligned}
-\int_{Q} \theta \frac{\partial \varphi}{\partial t} d x d t- & \int_{Q} u_{i} \theta \frac{\partial \varphi}{\partial x_{i}} d x d t+\int_{Q} \kappa(\theta) \nabla \theta \cdot \nabla \varphi d x d t \\
& =\int_{\Omega} \theta_{0}(x) \varphi(x, 0) d x+\int_{\bar{Q}} \varphi d \mu_{0} \forall \varphi \in C^{1}(\bar{Q}), \varphi(\cdot, T)=0 .
\end{aligned}
$$

On the other hand, observing that

$$
\liminf _{\varepsilon \rightarrow 0} \int_{Q} \nu\left(\theta_{\varepsilon}\right) D\left(\mathbf{u}_{\varepsilon}, \mathbf{u}_{\varepsilon}\right) \varphi d x d t \geq \int_{Q} \nu(\theta) D(\mathbf{u}, \mathbf{u}) \varphi d x d t \forall \varphi \in C(\bar{Q}), \varphi \geq 0
$$

it follows that

$$
\int_{\bar{Q}} \varphi d \mu_{0} \geq \int_{Q} \nu(\theta) D(\mathbf{u}, \mathbf{u}) \varphi d x d t \forall \varphi \in C^{1}(\bar{Q}), \varphi(\cdot, T)=0, \varphi \geq 0 .
$$

Now, for Borel sets $E \subseteq \bar{Q}$, define

$$
\mu(E):=\mu_{0}(E)-\int_{E} \nu(\theta) D(\mathbf{u}, \mathbf{u}) d x d t .
$$


We obtain

(i) $\mu(\emptyset)=0$,

(ii) $\mu\left(\bigcup_{i=1}^{\infty} E_{i}\right)=\sum_{i=1}^{\infty} \mu\left(E_{i}\right), E_{i} \subset \bar{Q}$ Borel, disjoint,

(iii) $\mu(E) \geq 0 \forall E \subseteq \bar{Q}$ Borel.

Here, (i) and (ii) are obvious, whereas (iii) follows from (3.11). Thus, $\mu \in \mathcal{M}(\bar{Q})$ and

$$
\int_{E} \varphi d \mu=\int_{E} \varphi d \mu_{0}-\int_{E} \nu(\theta) D(\mathbf{u}, \mathbf{u}) \varphi d x d \mu d t
$$

for any Borel set $E \subseteq \bar{Q}$ and any $\varphi \in C(\bar{Q})$. Now, (3.9) takes the form (3.3).

Finally, (3.6) is readily deduced from (2.9) and (2.10) by routine arguments.

Interpretation of (3.3). We identify the measure $\mu$ with a distribution in $Q$. Then (3.3) implies

$$
\frac{\partial \theta}{\partial t}+\mathbf{u} \cdot \nabla \theta=\nu(\theta) D(\mathbf{u}, \mathbf{u})-\nabla \cdot(\kappa(\theta) \nabla \theta)+\mu \quad \text { in } \mathcal{D}^{\prime}(Q) .
$$

This equation represents the difference (or defect) between the conservation of internal energy in the classical context (cf. (1.3)) and its counterpart in the context of distributions. The measure $\mu$ which may be called the defect measure, can be interpreted as an additional dissipation term which arises from discontinuities of the weak solution $\{\mathbf{u}, \theta\}$ of (1.1)-(1.5) under consideration.

The appearance of defect measures is a well-known phenomenon of limits of approximate solutions for nonlinear PDE's (cf. e.g., [1],[3], [6], [8], [9]).

4. Some properties of the defect measure $\mu$. Let $\left\{\mathbf{u}_{\varepsilon}, \theta_{\varepsilon}\right\}$ be an approximate solution to (1.1)-(1.5) obtained in Theorem 1 with initial data $\mathbf{u}_{0 \varepsilon} \in V_{6}, \theta_{0 \varepsilon} \in W^{1,2}$ such that $\mathbf{u}_{0 \varepsilon} \rightarrow \mathbf{u}_{0}$ strongly in $H, \theta_{0 \varepsilon} \rightarrow \theta_{0}$ strongly in $L^{1}$ as $\varepsilon \rightarrow 0$. Let $\{\mathbf{u}, \theta\}$ denote the limit of a convergent subsequence of $\left\{\mathbf{u}_{\varepsilon}, \theta_{\varepsilon}\right\}$ (cf. the proof of Theorem 2), and let $\mu_{0} \in \mathcal{M}(\bar{Q})$ be a Radon measure associated with $\left\{\mathbf{u}_{\varepsilon}, \theta_{\varepsilon}\right\}$ according to (3.8).

From the strong convergence $\mathbf{u}_{\varepsilon} \rightarrow \mathbf{u}$ in $\left[L^{2}(Q)\right]^{3}$ it follows that there exists a null-set $\mathcal{N} \subset] 0, T[$ such that

$$
\left.\lim _{\varepsilon \rightarrow 0}\left\|\mathbf{u}_{\varepsilon}(t)\right\|=\|\mathbf{u}(t)\| \forall t \in\right] 0, T[\backslash \mathcal{N} .
$$

We now prove the following

Proposition. For all $t \in] 0, T[\backslash \mathcal{N}$,

$$
\lim _{\varepsilon \rightarrow 0} \int_{0}^{t} \int_{\Omega} \nu\left(\theta_{\varepsilon}\right) D\left(\mathbf{u}_{\varepsilon}, \mathbf{u}_{\varepsilon}\right) \varphi d x d s=\int_{\bar{\Omega} \times[0, t]} \varphi d \mu_{0} \forall \varphi \in C(\bar{Q}) .
$$

Proof. Let $t \in] 0, T\left[\backslash \mathcal{N}\right.$. Let $\left.\left(t_{k}\right) \subset\right] 0, T\left[\backslash \mathcal{N}\right.$ be a sequence such that $t_{k}>t$ for all $k \in \mathbf{N}$, and $\lim _{k \rightarrow \infty} t_{k}=t$. For every $k \in \mathbf{N}$, take $\zeta_{k} \in C([0, T])$ satisfying

$$
0 \leq \zeta_{k} \leq 1 \text { in }[0, T], \quad \zeta_{k}(s)= \begin{cases}1 & \text { if } 0 \leq s \leq t \\ 0 & \text { if } t_{k} \leq s \leq T\end{cases}
$$


Let $\varphi \in C(\bar{Q})$ and $k \in \mathbf{N}$. We have

$$
\begin{aligned}
& \int_{0}^{t} \int_{\Omega} \nu\left(\theta_{\varepsilon}\right) D\left(\mathbf{u}_{\varepsilon}, \mathbf{u}_{\varepsilon}\right) \varphi d x d s-\int_{\bar{\Omega} \times[0, t]} \varphi d \mu_{0} \\
&=\int_{Q} \nu\left(\theta_{\varepsilon}\right) D\left(\mathbf{u}_{\varepsilon}, \mathbf{u}_{\varepsilon}\right) \varphi \zeta_{k} d x d s-\int_{\bar{Q}} \varphi \zeta_{k} d \mu_{0} \\
&-\int_{t}^{t_{k}} \int_{\Omega} \nu\left(\theta_{\varepsilon}\right) D\left(\mathbf{u}_{\varepsilon}, \mathbf{u}_{\varepsilon}\right) \varphi \zeta_{k} d x d s+\int_{\bar{\Omega} \times] t, t_{k}[} \varphi \zeta_{k} d \mu_{0} .
\end{aligned}
$$

Observing that

$$
\frac{1}{2}\left\|\mathbf{u}_{\varepsilon}\left(t_{k}\right)\right\|_{H}^{2}+\int_{t}^{t_{k}} \int_{\Omega} \nu\left(\theta_{\varepsilon}\right) D\left(\mathbf{u}_{\varepsilon}, \mathbf{u}_{\varepsilon}\right) d x d s=\frac{1}{2}\left\|\mathbf{u}_{\varepsilon}(t)\right\|_{H}^{2}+\int_{t}^{t_{k}}\left\langle\mathbf{f}, \mathbf{u}_{\varepsilon}\right\rangle_{V_{2}} d s
$$

(cf. (2.7)), we find

$$
\begin{aligned}
&\left|-\int_{t}^{t_{k}} \int_{\Omega} \nu\left(\theta_{\varepsilon}\right) D\left(\mathbf{u}_{\varepsilon}, \mathbf{u}_{\varepsilon}\right) \varphi \zeta_{k} d x d s\right| \leq \max _{\bar{Q}}|\varphi| \int_{t}^{t_{k}} \int_{\Omega} \nu\left(\theta_{\varepsilon}\right) D\left(\mathbf{u}_{\varepsilon}, \mathbf{u}_{\varepsilon}\right) d x d s \\
&=\max _{\bar{Q}}|\varphi|\left\{\frac{1}{2}\left(\left\|\mathbf{u}_{\varepsilon}(t)\right\|_{H}^{2}-\left\|\mathbf{u}_{\varepsilon}\left(t_{k}\right)\right\|_{H}^{2}\right)+\int_{t}^{t_{k}}\left\langle\mathbf{f}, \mathbf{u}_{\varepsilon}\right\rangle_{V_{2}} d x\right\} .
\end{aligned}
$$

Thus, by (3.8) and (4.1),

$$
\begin{aligned}
& \limsup _{\varepsilon \rightarrow 0}\left|\int_{0}^{t} \int_{\Omega} \nu\left(\theta_{\varepsilon}\right) D\left(\mathbf{u}_{\varepsilon}, \mathbf{u}_{\varepsilon}\right) \varphi d x d s-\int_{\bar{\Omega} \times[0, t]} \varphi d \mu_{0}\right| \\
\leq & \max _{\bar{Q}}|\varphi|\left\{\frac{1}{2}\left(\|\tilde{\mathbf{u}}(t)\|_{H}^{2}-\left\|\tilde{\mathbf{u}}\left(t_{k}\right)\right\|_{H}^{2}\right)+\int_{t}^{t_{k}}\langle\mathbf{f}, \mathbf{u}\rangle_{V_{2}} d s\right\}+\max _{\bar{Q}}|\varphi| \mu_{0}(\bar{\Omega} \times] t, t_{k}[),
\end{aligned}
$$

where $\tilde{\mathbf{u}} \in \mathbf{u}$ denotes the representative belonging to $C_{w}([0, T] ; H)$.

Clearly,

$$
\limsup _{k \rightarrow \infty}\left(\|\tilde{\mathbf{u}}(t)\|_{H}^{2}-\left\|\tilde{\mathbf{u}}\left(t_{k}\right)\right\|_{H}^{2}\right) \leq 0, \lim _{k \rightarrow \infty} \mu_{0}(\bar{\Omega} \times] t, t_{k}[)=\mu_{0}\left(\bigcap_{k=1}^{\infty}(\bar{\Omega} \times] t, t_{k}[)\right)=0 .
$$

Taking the $\lim \sup _{k \rightarrow \infty}$ on both sides of (4.4) gives the claim.

To proceed, we note that (2.5) implies

$$
\begin{aligned}
\int_{\Omega} \theta_{\varepsilon}(x, t) \eta(x) d x-\int_{0}^{t} \int_{\Omega} u_{\varepsilon i} \theta_{\varepsilon} & \frac{\partial \eta}{\partial x_{i}} d x d s+\int_{0}^{t} \int_{\Omega} \kappa\left(\theta_{\varepsilon}\right) \nabla \theta_{\varepsilon} \cdot \nabla \eta d x d s \\
= & \int_{\Omega} \theta_{0 \varepsilon}(x) \eta(x) d x+\int_{0}^{t} \int_{\Omega} \nu\left(\theta_{\varepsilon}\right) D\left(\mathbf{u}_{\varepsilon}, \mathbf{u}_{\varepsilon}\right) \eta d x d s
\end{aligned}
$$

for a.e. $t \in[0, T]$ and all $\eta \in W^{1, \rho}(\rho=8 r /(5 r-6)$ with $6 / 5<r<5 / 4)$. Observing (4.2), the passage to the limit $\varepsilon \rightarrow 0$ gives, for any $\eta \in W^{1, \rho}$,

$$
\begin{aligned}
\int_{\Omega} \theta(x, t) \eta(x) d x & -\int_{0}^{t} \int_{\Omega} u_{i} \theta \frac{\partial \eta}{\partial x_{i}} d x d s+\int_{0}^{t} \int_{\Omega} \kappa(\theta) \nabla \theta \cdot \nabla \eta d x d s \\
= & \int_{\Omega} \theta_{0}(x) \eta(x) d x+\int_{0}^{t} \int_{\Omega} \nu(\theta) D(\mathbf{u}, \mathbf{u}) \eta d x d s+\int_{\bar{\Omega} \times[0, T]} \eta d \mu
\end{aligned}
$$

for a.e. $t \in[0, T]$ ( $\mu$ according to (3.11)). Here, by virtue of the separability of $W^{1, \rho}$, the null-set in $[0, T]$ where the equation in (4.5) fails does not depend on $\eta$. 
Thus, there exists a null-set $\left.\mathcal{N}_{0} \subset\right] 0, T$ [ such that (4.1) and the equation in (4.5) hold for all $t \in] 0, T\left[\backslash \mathcal{N}_{0}\right.$, and

$$
\begin{aligned}
& \int_{\Omega}\left(\frac{1}{2}|\mathbf{u}(x, t)|^{2}+\theta(x, t)\right) d x \\
&\left.=\int_{\Omega}\left(\frac{1}{2}\left|\mathbf{u}_{0}(x)\right|^{2}+\theta_{0}(x)\right) d x+\int_{0}^{t}\langle\mathbf{f}, \mathbf{u}\rangle_{V_{2}} d s \forall t \in\right] 0, T\left[\backslash \mathcal{N}_{0}\right.
\end{aligned}
$$

We now prove

TheOrem 3. Let $\mathcal{N}_{0}$ be as above. Then:

(i) For all $\left.t, t^{\prime} \in\right] 0, T\left[\backslash \mathcal{N}_{0}, t<t^{\prime}\right.$,

$$
\begin{aligned}
\frac{1}{2} \int_{\Omega}\left|\mathbf{u}\left(t^{\prime}\right)\right|^{2} d x+\int_{t}^{t^{\prime}} \int_{\Omega} \nu(\theta) D(\mathbf{u}, \mathbf{u}) d x d s+ & \left.\left.\mu(\bar{\Omega} \times] t, t^{\prime}\right]\right) \\
& =\frac{1}{2} \int_{\Omega}|\mathbf{u}(t)|^{2} d x+\int_{t}^{t^{\prime}}\langle\mathbf{f}, \mathbf{u}\rangle_{V_{2}} d s
\end{aligned}
$$

(ii) $\mu(\bar{\Omega} \times\{0\})=0$;

(iii) $\mu(\bar{\Omega} \times\{t\})>0$ for at most countably many $t \in] 0, T[$;

(iv) fix $6 / 5<r<5 / 4$, define $\rho:=8 r /(5 r-6)$; then

$$
\begin{aligned}
& \left\|\theta\left(t^{\prime}\right)-\theta(t)\right\|_{\left(W^{1, \rho}\right)^{*}} \\
& \left.\left.\quad \leq c\left\{\int_{t}^{t^{\prime}}\left(\|\nabla \theta(s)\|_{L^{r}}+\|u(s)\|_{L^{8 / 3}}\|\theta(s)\|_{L^{4 / 3}}+\|\nabla u(s)\|_{L^{2}}^{2}\right) d s+\mu(\bar{\Omega} \times] t, t^{\prime}\right]\right)\right\}
\end{aligned}
$$

for all $\left.t, t^{\prime} \in\right] 0, T\left[\backslash \mathcal{N}_{0}, t<t^{\prime}\right.$.

Proof. (i) Take $\eta=1$ in (4.5). Combining this with (4.6) implies

$$
\begin{aligned}
& \frac{1}{2} \int_{\Omega}|\mathbf{u}(t)|^{2} d x+\int_{0}^{t} \int_{\Omega} \nu(\theta) D(\mathbf{u}, \mathbf{u}) d x d s+\mu(\bar{\Omega} \times[0, t]) \\
& =\frac{1}{2} \int_{\Omega}|\mathbf{u}(t)|^{2} d x+\int_{\Omega} \theta(t) d x-\int_{\Omega} \theta_{0} d x=\frac{1}{2} \int_{\Omega}\left|\mathbf{u}_{0}\right|^{2} d x+\int_{0}^{t}\langle\mathbf{f}, \mathbf{u}\rangle_{V_{2}} d s .
\end{aligned}
$$

This equation also holds with $t^{\prime}$ in place of $t$. Subtracting the two equations gives the desired result.

(ii) Let $\left.\left(\tau_{k}\right) \subset\right] 0, T\left[\backslash \mathcal{N}_{0}\right.$ be a sequence such that $\lim _{k \rightarrow \infty} \tau_{k}=0$. Again using (4.5) with $\eta=1$, and (4.6) gives

$$
\begin{aligned}
\mu(\bar{\Omega} \times\{0\}) & =\lim _{k \rightarrow \infty} \mu\left(\bar{\Omega} \times\left[0, \tau_{k}\right]\right) \\
& =\lim _{k \rightarrow \infty}\left(\int_{\Omega} \theta\left(\tau_{k}\right) d x-\int_{\Omega} \theta_{0} d x-\int_{0}^{\tau_{k}} \int_{\Omega} \nu(\theta) D(\mathbf{u}, \mathbf{u}) d x d s\right)=0 .
\end{aligned}
$$

(iii) The claim follows immediately from $\mu(\bar{Q}) \leq \mu_{0}(\bar{Q})<\infty$. 
(iv) From (4.5) it follows that, for any $\eta \in W^{1, \rho}$,

$$
\begin{aligned}
& \left|\int_{\Omega}\left(\theta\left(x, t^{\prime}\right)-\theta(x, t)\right) \eta(x) d x\right| \\
& \left.\left.\quad \leq \int_{t}^{t^{\prime}}\left|\left(-\kappa(\theta) \nabla \theta \cdot \nabla \eta+u_{i} \theta \frac{\partial \eta}{\partial x_{i}}+\nu(\theta) D(\mathbf{u}, \mathbf{u}) \eta\right) d x\right| d s+\max _{\bar{\Omega}}|\eta| \mu(\bar{\Omega} \times] t, t^{\prime}\right]\right) \\
& \left.\left.\quad \leq c\left\{\int_{t}^{t^{\prime}}\left(\|\nabla \theta(s)\|_{L^{r}}+\|\mathbf{u}(s)\|_{L^{8 / 3}}\|\theta(s)\|_{L^{4 / 3}}+\|\nabla \mathbf{u}(s)\|_{L^{2}}^{2}\right) d s+\mu(\bar{\Omega} \times] t, t^{\prime}\right]\right)\right\}\|\nabla \eta\|_{L^{\rho}}
\end{aligned}
$$

(cf. [12] for details), whence the claim.

REMARK. The term $\left.\left.\mu(\bar{\Omega} \times] t, t^{\prime}\right]\right)$ in Theorem 3 (i) may be viewed as the "missing term" for the energy equality for weak solutions to the Navier-Stokes equations. By an entirely different argument, a dissipation term in a local form of the energy equality for the NavierStokes equations has been obtained in [2]. A refinement of the usual energy inequality for weak solutions to these equations has been established in [10], [11].

\section{References}

[1] R. Alexandre and C. Villani, On the Boltzmann equation for long-range interactions, Comm. Pure Appl. Math. 55 (2002), 30-70.

[2] J. Duchon and R. Robert, Initial energy dissipation for weak solutions of incompressible Euler and Navier-Stokes equations, Nonlinearity 13 (2000), 249-255.

[3] E. Feireisl, Dynamics of Compressible Flow, Oxford Sci. Publ., Clarendon Press Oxford, 2003.

[4] - Mathematical theory of compressible, viscous and heat-conducting fluids, to appear.

[5] G. Gallavotti, Foundations of Fluid Dynamics, Springer-Verlag, Berlin, 2002.

[6] P. Harpes, Bubbling of approximations for the 2-D Landau-Lifschitz flow, Comm. Partial Diff. Equ. 31 (2006), 1-20.

[7] L. D. Landau and E. M. Lifschitz, Lehrbuch der theoretischen Physik, Bd. VI: Hydrodynamik, 3. Aufl., Akademie-Verlag, Berlin, 1978.

[8] R. Lewandowski, The mathematical analysis of the coupling of a turbulent kinetic energy equation to the Navier-Stokes equation with an eddy viscosity, Nonlin. Analysis 28 (1997), 393-417.

[9] F.-H. Lin, C. Liu and P. Zhang, On hydrodynamics of viscoelastic fluids, Comm. Pure Appl. Math. 58 (2006), 1437-1471.

[10] T. Nagasawa, A new energy inequality and partial regularity for weak solutions of NavierStokes equations, J. Math. Fluid Mech. 3 (2001), 40-56.

[11] - A refinement of the energy inequality for the Navier-Stokes equations, Nonlinear Anal. 47 (2001), 4245-4256.

[12] J. Naumann, On the existence of weak solutions to the equations on non-stationary motion of heat-conducting incompressible viscous fluids, Math. Meth. Appl. Sci. 29 (2006), 1883-1906.

[13] - An existence theorem for weak solutions to the equations of non-stationary motion of heat-conducting incompressible viscous fluids, J. Nonlin. Convex Anal. 7 (2006), 483-497.

[14] A. Novotný and I. Straškraba, Introduction to the Mathematical Theory of Compressible Flow, Oxford Univ. Press, New York, 2004. 